\title{
Twitter como plataforma de los alcaldes para la comunicación pública
}

\author{
Beatriz CATALINA GARCÍA \\ Universidad Rey Juan Carlos de Madrid \\ beatriz.catalina@urjc.es \\ Ma Cruz López de Ayala López \\ Universidad Rey Juan Carlos de Madrid \\ mariacruz.lopezdeayala@urjc.es \\ José Gabriel Fernández FernándeZ \\ Universidad Rey Juan Carlos de Madrid \\ josegabriel.fernandez@urjc.es
}

Recibido: 31 de julio de 2014

Aceptado: 5 de febrero de 2015

\section{Resumen}

En el ámbito de un creciente escepticismo político, Twitter constituye una alternativa para potenciar la comunicación con los ciudadanos e implicarlos en la agenda municipal. Mediante un seguimiento y análisis de contenido de los perfiles abiertos en Twitter por los alcaldes de capitales de provincia y presidentes de ciudades autonómicas de España, el objetivo del artículo es examinar el uso que se hace de esta herramienta de microblogging y las posibilidades de participación que se abren a la ciudadanía. El estudio concluye que los alcaldes no están utilizando adecuadamente sus cuentas para favorecer el diálogo con sus representados: hacen un uso escaso, las temáticas abordadas no son las que más interesan a sus representados y las respuestas a las inquietudes mostradas por los usuarios en sus comentarios resulta muy limitada.
\end{abstract}

Palabras clave: Twitter, participación ciudadana, administración municipal, interactividad.

\section{The use of Twitter by mayors to promote public communications}

\begin{abstract}
In the context of an increasing political skepticism, Twitter is an alternative to promote communication with citizens and to involve them in the municipal agenda. Through monitoring and content analysis of the profiles opened in Twitter by the mayors of provincial capitals and presidents of autonomous cities of Spain, the aim of the article is to examine how they are making use of this microblogging site and the possibilities of participation that are opened for the citizenship. The study concludes that the mayors are not properly using their accounts in order to promote dialogue with their represented ones: they make a scarce use, the topics addressed are not those that their represented one are most interested and their answers to the concerns shown by users in their comments are very limited.
\end{abstract}

Keywords: Twitter, civil participation, municipal administration, interactivity.

\section{Referencia normalizada}

CATALINA GARCÍA, Beatriz; LÓPEZ DE AYALA LÓPEZ, Ma Cruz; y FERNÁNDEZ FERNÁNDEZ, José Gabriel (2015): “Twitter como plataforma de los alcaldes para la comunicación pública”. Estudios sobre el Mensaje Periodístico. Vol. 21, Núm. 2 (julio-diciembre), págs.: 757-772. Madrid, Servicio de Publicaciones de la Universidad Complutense.

Sumario: 1. Introducción. 2. Marco teórico. 3. Objetivos. 4. Metodología; 4.1. Variables utilizadas en el estudio. 5. Resultados. 6. Conclusiones y discusión. 7. Referencias bibliográficas. 


\section{Introducción}

Queda fuera de toda duda que los gobiernos locales constituyen el modelo de representación más cercano a los ciudadanos; una idea ya expuesta a finales del XIX por J.S. Mill y corroborada hasta la actualidad por gran cantidad de autores (Janoschka, 2011; Brugué et al., 2005; Pindado, 2004; Font y Parrado, 2000). Tampoco se pone en tela de juicio que la participación de la ciudadanía se considera un elemento central de una democracia saludable.

Aunque suponen un claro avance en la participación, las fórmulas actualmente reglamentadas en gran parte de los municipios (principalmente en los de mayor espectro poblacional) y configuradas en consejos sectoriales, comités ciudadanos o con terminología similar, no han sido plenamente eficaces para que los particulares mantengan un contacto directo y constante con sus representantes políticos. En este contexto, la emergencia de los nuevos medios sociales que facilitan la comunicación bidireccional ha atraído la atención de los investigadores en cuanto que son herramientas que potencialmente pueden incitar a la implicación activa del ciudadano en lo público y en lo político.

En particular, Internet, y más concretamente, las redes sociales constituyen una importante vía de acceso para la comunicación sin protocolos entre los representantes públicos y la ciudadanía, haciendo posible relacionar de forma directa las necesidades de los ciudadanos y las decisiones de la autoridad representante. En el marco de la administración local, esta alternativa tiene un valor económico añadido: resulta un medio asequible que no requiere apenas costes para unas arcas municipales que, desde que se configuró el actual mapa autonómico y de transferencias competenciales en el Estado español, adolecen de problemas de financiación que se han visto agravados en los últimos años por la crisis que afecta a todos los niveles administrativos y sociales.

Twitter constituye una red social que, como ya se verá más adelante, actúa como principal referente de los políticos pertenecientes a los distintos ámbitos territoriales que exponen de modo abierto y público sus planteamientos, propuestas o ideas con la posibilidad de que sean respondidas o ampliadas sin filtros por cualquier ciudadano que esté situado en el lado positivo de la brecha digital.

Aunque proliferan las investigaciones enfocadas, desde diversos puntos de vista, sobre la comunicación política en Twitter (Kruikemeier, 2014; Mancera y Pano, 2013; Deltell et al., 2012; Fernández, 2012; Congosto, 2011; Tumasjan, 2010), la literatura científica que centra su atención en los representantes municipales y su participación en las redes sociales resulta escasa. Con el objetivo de superar esta carencia y profundizar en el grado de interactividad que mantienen los representantes públicos con sus representados y el grado de implicación comunicacional de estos últimos, este trabajo analiza el uso que los alcaldes de las capitales de provincia y presidentes de ciudades autonómicas y la ciudadanía hacen de Twitter.

\section{Marco teórico}

La defensa de la participación ciudadana en la agenda política de los sistemas democráticos ha sido profusamente tratada en el ámbito científico (Brugué et al., 2005; Pas- 
tor Seller, 2009; Crawford, 2009; Gonzálvez y Contreras Pulido, 2011). En este contexto, Dalhgren (2005) en clara referencia a las teorías promulgadas a principios del siglo XX por Habermas y Denwey, afirma que no se debe olvidar que el concepto de la democracia reside en las conversaciones que los ciudadanos entablan con el resto.

No solo la literatura científica insiste en la importancia de la implicación activa ciudadana, también organismos públicos de distinto ámbito territorial se han ocupado de esta cuestión, especialmente desde la primera década del siglo XXI. La Constitución Española de 1978, en su artículo 9.2 sobre Libertad e Igualdad, señala que a los poderes públicos les corresponde "facilitar la participación de todos los ciudadanos en la vida política, económica, cultural y social"; aspecto que también se remarca en el artículo 129.1 Otros ejemplos claros y centrados en la administración municipal se encuentran en la Recomendación sobre este asunto elaborada por el Consejo de Ministros del Consejo de Europa (2001), en el Libro Blanco para la Administración Local publicado por el Ministerio de Administraciones Públicas (2005) y en la legislación autonómica de régimen local, aunque en este caso con escasa relevancia, en contraste con la Ley de Regulación de Bases del Régimen Local (1985) que, en varios de sus artículos, detalla expresamente el derecho a participar en la gestión municipal, las técnicas dirigidas a la información y participación ciudadana y el mandato a las corporaciones locales para que faciliten dicha participación entre todos los ciudadanos. No obstante, esta Ley subordina la participación ciudadana a la participación política la cual corresponde a los órganos representativos.

Si bien hay que reseñar algunas voces reticentes a una amplia implicación ciudadana objetivada durante los últimos años en las aplicaciones ofrecidas por Internet. En este marco, Torres da Silva (2013) asegura que una discusión idónea no surge de forma automática por una mayor participación, sino que puede traer como consecuencia el llamado trolling, término con el que la autora engloba a las publicaciones destinadas a desinformar, engañar o trivializar el debate. Culver \& Jacobson (2012) apuestan por una previa alfabetización mediática para fomentar la participación cívica; Havick (2000) muestra su preocupación por la fragmentación de la información en Internet ya que, a su juicio, constituye un problema para la deliberación; mientras que Margolis y Harnick (2000) niegan que la información aportada en Internet constituye un factor de transformación social.

Sin embargo, es evidente la creciente implantación y el continuo ascenso de espacios on line, principalmente las redes sociales, que facilitan, entre otras aplicaciones, la comunicación de tú a tú, sin jerarquías, entre los ciudadanos y los representantes políticos. La encuesta anual "Navegantes en la Red" (AIMC, 2013) confirma la cotidianeidad asumida por un $67,4 \%$ de los internautas que se conecta a las redes sociales diariamente, frente al 43,5\% que lo hacía en el 2009 y el 28,6\% detectado en el 2008. El mismo estudio destaca el liderazgo indiscutible de Facebook, con un 90\% de internautas, mientras que el crecimiento de Twitter apenas ha sido perceptible, tan solo de un $1 \%$, lo que la sitúa en un $45 \%$ de usuarios de las redes sociales, aunque mantiene su segundo puesto. Varona (2014) pone igualmente de manifiesto la preeminencia en España de Facebook sobre Twitter, no solo por la cantidad de usuarios, sino también por la actividad que muestran en sus cuentas. 
El "IV Estudio Anual de Redes Sociales" (IAB, 2013) registra datos similares: el $80 \%$ de usuarios de Internet utiliza las redes sociales, siendo Facebook la más popular, seguida por Twitter. No obstante, según los datos extraídos por Castelló et al. (2014) de la empresa Com Store, esta red social experimentó en España entre noviembre del 2009 y noviembre del 2010 un incremento en el número de usuarios de un $151 \%$, lo que le convirtió en el país con mayor crecimiento.

Aunque la utilidad y efectividad de Twitter es puesta en tela de juicio por diversos autores (Arroyo, 2012; Metaxas et al., 2011), numerosas investigaciones aluden a la utilización de Twitter y las ventajas que ello conlleva (Bollen et al., 2011; Romero et al., Meeder \& Kleinberg, 2011; Pak \& Paroubek, 2010; Java et al., 2007). Rodríguez Andrés y Ureña (2011), además de destacar la preponderancia de Twitter en el ámbito periodístico y político, afirman, entre otros argumentos para justificar su uso, la facilidad que entraña para conversar con los ciudadanos, a los cuales les convierte en "líderes de opinión de su entorno" al tiempo que los políticos se "humanizan y aumenta la empatía hacia ellos".

Finalmente, en cuanto a su forma de utilización, Caldevilla (2009) puntualiza que no es tan importante una ingente recopilación de seguidores como la necesidad de conocer y comprender la red para obtener de ella un mejor y mayor provecho. En el ámbito electoral, Ammann (2010) concluye que Twitter se ha convertido en una importante plataforma para los candidatos; mientras que Barberá y Rivero (2011), en su estudio sobre esta red durante las elecciones legislativas españolas del 2011, destacan la importancia de los retuits (informaciones elaboradas por un usuario y que son compartidas por otros). Castelló et al. (2014), aunque en referencia al ámbito corporativo y de la publicidad, también destacan la importancia de participación de los usuarios y el contacto continuo entre éstos y con éstos; y, en la misma línea, Crawford (2009), desde la generalidad de Internet como instrumento de la democracia deliberativa, asegura que los comentarios no deben quedarse aislados, sino que es deseable que se lean, internalicen y se respondan por otros individuos.

\section{Objetivos}

En el marco del debate sobre las potencialidades democratizadoras de las herramientas de comunicación interactiva que ofrece Internet, el propósito de este artículo es conocer el uso que se está haciendo de Twitter en el ámbito de la administración municipal. Los objetivos del artículo se mueven en torno a dos ejes: por un lado, describir si los máximos representantes públicos acuden a este recurso de microblogging para comunicarse con sus representados y sus formas de uso, haciendo hincapié en el aprovechamiento de las potencialidades de comunicación interactiva que Twitter ofrece para favorecer la participación activa e involucración de la ciudadanía en la toma de decisiones de su localidad. Por otro lado, se trata de aproximarnos al uso efectivo que los ciudadanos están haciendo de estas herramientas que los gobernantes locales ponen a su disposición, analizando el grado y formas de participación.

Con esta finalidad, se ha examinado si los alcaldes de capitales de provincias y ciudades autonómicas tienen activa una cuenta de Twitter, el número de seguidores, la frecuencia de tuits públicos, la temática de esos tuits y retuits, el uso de otras he- 
rramientas de comunicación multimedia específicas que Twitter ofrece para poder interactuar con otros -vídeos, fotos-, así como la contestación de la ciudadanía a estas propuestas temáticas y las respuestas, a su vez, de los propios alcaldes a estas formas participativas.

\section{Metodología}

Con el objetivo de acotar el universo de estudio de nuestra investigación, se seleccionaron a los cincuenta alcaldes de capitales de provincia de las diecisiete comunidades autónomas y los dos presidentes de las ciudades autónomas de Ceuta y Melilla.

En una primera etapa, se realizó un seguimiento en Twitter con el propósito de localizar las cuentas activas durante el mes de mayo de 2014 de la totalidad de nuestra población. De los 52 alcaldes de capitales de provincia y presidentes de ciudades autónomas, sólo 39 mantienen cuentas abiertas en Twitter en el periodo que cubre nuestro análisis. Es necesario precisar que nuestro objetivo era identificar las cuentas activas asociadas al rol de máxima autoridad política de la administración municipal y no como personas particulares. A este respecto, cabe mencionar la identificación de una cuenta que fue abierta por uno de los alcaldes que conforman nuestro universo de estudio previamente a su nombramiento, desapareciendo prácticamente la actividad coincidiendo con la toma de posesión de este cargo; esta cuenta fue desechada en el análisis.

Por otra parte, el criterio para clasificar una cuenta como inactiva ha sido que ésta no hubiera cesado su actividad durante al menos tres meses previos al periodo que abarca nuestro análisis. Nueve de las treinta y ocho cuentas identificadas se encuentran en esta situación. En definitiva, se han localizado 29 cuentas activas.

En una segunda etapa, se realizó un análisis de contenido de los perfiles de cada una de las cuentas y su actividad desde el 1 al 31 de mayo de 2014. Los datos resultantes han sido codificados y registrados manualmente en una base de datos. Una incidencia destacable hace referencia a la localización de dos cuentas que, cumpliendo el criterio establecido para definirla como activa, experimentaron un parón en su actividad coincidiendo con el periodo de nuestro análisis. Aunque se han considerado estas cuentas como activas a efectos de contabilización del uso de esta herramienta, no obstante, y como resulta obvio, no se han incluido en el análisis de los perfiles y actividad a lo largo del periodo de estudio.

Otro aspecto destacable es la coincidencia del universo temporal objeto de estudio con las elecciones europeas, lo que podría condicionar los resultados del trabajo al generar un incremento inusual de la actividad en Twitter. Debido a esta incidencia, se decidió incluir un ítem específico que registrara este aspecto entre los temas abordados en los tuits analizados.

Recopilando brevemente los datos sobre la población analizada, se identificaron treinta y ocho cuentas activas de alcaldes de capitales de provincia y presidentes de ciudades autónomas en su rol de máximos representantes locales, de las cuales únicamente veintinueve permanecen activas y veintisiete mantuvieron, además, la actividad durante el universo temporal que cubre nuestro estudio: el mes de mayo de 2014. 
El perfil y contenidos de todas las cuentas están en castellano, a excepción de las cuatro capitales de provincia catalanas y Orense y Pontevedra en la Comunidad de Galicia, que utilizaban la lengua cooficial específica de su comunidad.

\subsection{Variables utilizadas en el estudio}

- Número de seguidores: número total de personas que siguen la cuenta.

- Frecuencia de tuits. Los tuits (tweets en inglés) son mensajes de texto, de longitud máxima de 140 caracteres, que se muestran en la página principal del usuario. Se registra el número total de mensajes enviados a lo largo del mes de mayo y el promedio por alcalde y dia.

- Retuits: mensajes que los alcaldes remiten de otras cuentas para difundirlos entre sus propios seguidores.

- Retuiteados: mensajes que los seguidores de las cuentas analizadas postean para compartir con sus propios seguidores, contribuyendo a difundir los mensajes de los alcaldes.

- Posibilidades multimedia, con el objetivo de medir la profundización del uso que los alcaldes hacen de Twitter para interactuar con sus representados, se ha considerado también el uso que los alcaldes hacen de diferentes posibilidades multimedia, en particular: videos y fotos.

- Temática. Los tuits se han clasificado siguiendo una tipología de nueve ítems según la temática tratada. A continuación se incluye una breve descripción de cada uno de estos ítems.

- Cultura: referencias a música, exposiciones, fiestas patronales, tradicionales y de diferentes ámbitos, premios de diferentes tendencias culturales y otros temas culturales.

- Deportes: comentarios de cualquier actividad deportiva, ya sea profesional como amateur.

- Economía: incluye mensajes relacionados con turismo, empleo, datos económicos, emprendedores, empresa, industria, comercio o agricultura.

- Educación: alusiones a cualquiera de los niveles educativos, desde infantil a universitario, y enseñanzas transversales.

- Elecciones europeas: informaciones sobre la campaña, propaganda, mítines, resultados o balance de las elecciones al Parlamento Europeo.

- Institucional: referencias a actos de agenda, visitas de los alcaldes a diferentes sedes, sesiones plenarias, visitas de ciudadanos y/o personalidades públicas a la ciudad o al Ayuntamiento.

- Local: obras, infraestructuras, movilidad y aquellos aspectos relacionados con los barrios e identificativos de la ciudad.

- Medio Ambiente: comentarios con referencias a ecología y desarrollo sostenible.

- Política: alusiones a actos de partido, críticas a partidos rivales o instituciones de otros ámbitos territoriales, así como iniciativas y proyectos para el municipio anunciados por el alcalde e incluidos en su agenda política.

- Tecnología: referencias a nuevas tecnologías e Investigación y Desarrollo. 
- Social: comentarios relativos a sanidad y salud, servicios sociales, solidaridad, homenajes, sucesos o problemas vecinales.

- Otros: otros temas no recogidos en las categorías anteriores.

- Interactividad, esta variable ha sido medida haciendo uso de dos indicadores que nos orientan sobre la generación de un verdadero diálogo entre los alcaldes y los ciudadanos a través de Twitter:

- Respuestas de los seguidores a los mensajes o tuits remitidos por los usuarios principales. Estos comentarios son recogidos bajo un mismo Hashtag o etiqueta que identifica un tema y vienen precedida por el símbolo \#. Estas respuestas se han clasificado en los diferentes ítems, no excluyentes: queja, queja/sugerencia, sugerencia, pregunta, comentarios positivos, comentarios neutros e información. La diferencia entre estos dos últimos reside en que mientras los comentarios neutros no aportan un mensaje complementario al tuit principal, la información sí ofrece un contenido añadido.

- Respuestas de los usuarios principales de las cuentas analizadas a los comentarios de los seguidores.

\section{Resultados}

De los cincuenta y dos alcaldes de capitales de provincia y presidentes de ciudades autónomas considerados en el estudio, se identificaron veintinueve cuentas activas, de las cuales únicamente veintisiete registraron actividad a lo largo de mayo de 2014.

En la tabla 1, se puede observar que el número de seguidores de estas cuentas varía notablemente, desde apenas ochocientos seguidores hasta más de cincuenta mil. No obstante, hay que considerar que estas variaciones pueden venir afectadas por el tiempo que la cuenta ha permanecido abierta, entre otros aspectos.

Se enviaron un total de 1.739 tuits desde las cuentas activas analizadas a lo largo del mes de mayo, con una amplitud de rango que oscila desde un mínimo de siete tuits hasta un valor máximo de 279 mensajes remitidos. El promedio de tuits publicados fue de 64,4 mensajes por cada alcalde y 56,1 al día. En conjunto, apenas se alcanza una media de dos tuits remitidos por alcalde y día.

De todos los tuits publicados por los alcaldes, 601 fueron retuits, es decir, más de un tercio $(34,5 \%)$ son mensajes que no son generados por los alcaldes sino que éstos postean o remiten de otras cuentas. En la tabla 1 se puede observar que, incluso, en algún caso todos los tuits son remitidos desde otras cuentas y, al contrario, también encontramos algún caso en el que el cien por cien son mensajes generados por el usuario principal.

Por otra parte, los tuits publicados por los alcaldes han sido retuiteados, y por tanto difundidos, en 10.585 nuevas cuentas. El rango de mensajes retuiteados, considerando todas las cuentas que los han difundido, oscila entre un mínimo de 0 hasta 4.278 por alcalde, llegando a alcanzar hasta 768 retuits de un mismo mensaje por parte de los usuarios. En conjunto, el 53,6\% de los mensajes han sido retuiteados por los ciudadanos, contribuyendo a su difusión. Sin embargo, aunque algunos alcaldes consiguen que el $100 \%$ de todos sus mensajes sean retuiteados por otros usuarios, otros no han logrado que ninguno de sus tuits se difunda más allá de su propia cuenta. 
Respecto al aprovechamiento de las posibilidades multimedia que ofrece Twitter, cabe señalar el uso moderado que se hace de la fotografía (670), frente al escaso recurso al vídeo (37).

Tabla 1. Número de seguidores, frecuencia de tuits, retuits, mensajes retuiteados, fotos y vídeos respecto al total de tuits enviados por los representantes municipales.

Fuente: elaboración propia.

\begin{tabular}{|c|c|c|c|c|c|c|}
\hline Alcaldes & $\begin{array}{l}\mathrm{N}^{\mathrm{a}} \text { de } \\
\text { seguidores }\end{array}$ & $\begin{array}{c}\text { Frecuencia } \\
\text { Tuits }\end{array}$ & $\begin{array}{c}\text { Retuits } \\
\text { que hacen } \\
\text { los alcaldes } \\
(\%)\end{array}$ & $\begin{array}{c}\text { Retuiteados } \\
\text { por otros } \\
\text { usuarios (\%) }\end{array}$ & $\begin{array}{c}\text { Fotos } \\
(\%)\end{array}$ & $\begin{array}{c}\text { Vídeos } \\
\text { (\%) }\end{array}$ \\
\hline 1 & 3986 & 44 & $0,00 \%$ & $93,2 \%$ & $59,09 \%$ & $0,00 \%$ \\
\hline 2 & 1519 & 92 & $26,09 \%$ & $58,70 \%$ & $60,87 \%$ & $0,00 \%$ \\
\hline 3 & 57836 & 279 & $31,90 \%$ & $62,7 \%$ & $20,43 \%$ & $4,66 \%$ \\
\hline 4 & 895 & 65 & $4,62 \%$ & $38,5 \%$ & $1,54 \%$ & $0,00 \%$ \\
\hline 5 & 2153 & 110 & $87,27 \%$ & $9,1 \%$ & $35,45 \%$ & $3,64 \%$ \\
\hline 6 & 2949 & 108 & $14,81 \%$ & $49,1 \%$ & $48,15 \%$ & $1,85 \%$ \\
\hline 7 & 10866 & 36 & $16,67 \%$ & $80,6 \%$ & $41,67 \%$ & $0,00 \%$ \\
\hline 8 & 1257 & 7 & $100,00 \%$ & $0,0 \%$ & $85,71 \%$ & $0,00 \%$ \\
\hline 9 & 4658 & 79 & $2,53 \%$ & $60,8 \%$ & $58,23 \%$ & $0,00 \%$ \\
\hline 10 & 4777 & 104 & $79,81 \%$ & $13,5 \%$ & $61,54 \%$ & $4,81 \%$ \\
\hline 11 & 9857 & 189 & $18,52 \%$ & $72,5 \%$ & $55,56 \%$ & $0,53 \%$ \\
\hline 12 & 3293 & 18 & $5,56 \%$ & $83,3 \%$ & $88,89 \%$ & $0,00 \%$ \\
\hline 13 & 17063 & 40 & $0,00 \%$ & $100,0 \%$ & $0,00 \%$ & $0,00 \%$ \\
\hline 14 & 1714 & 6 & $50,00 \%$ & $100,0 \%$ & $0,00 \%$ & $0,00 \%$ \\
\hline 15 & 846 & 24 & $29,17 \%$ & $83,3 \%$ & $50,00 \%$ & $0,00 \%$ \\
\hline 16 & 5681 & 69 & $71,01 \%$ & $98,6 \%$ & $33,33 \%$ & $0,00 \%$ \\
\hline 17 & 5075 & 41 & $41,46 \%$ & $90,2 \%$ & $24,39 \%$ & $2,44 \%$ \\
\hline 18 & 3524 & 7 & $0,00 \%$ & $100,0 \%$ & $14,29 \%$ & $14,29 \%$ \\
\hline 19 & 1038 & 44 & $47,73 \%$ & $88,6 \%$ & $25,00 \%$ & $4,55 \%$ \\
\hline 20 & 42701 & 66 & $3,03 \%$ & $100,0 \%$ & $31,82 \%$ & $7,58 \%$ \\
\hline 21 & 2013 & 45 & $82,22 \%$ & $93,3 \%$ & $60,00 \%$ & $0,00 \%$ \\
\hline 22 & 7030 & 123 & $25,20 \%$ & $86,2 \%$ & $26,02 \%$ & $0,81 \%$ \\
\hline 23 & 3498 & 48 & $64,58 \%$ & $100,0 \%$ & $22,92 \%$ & $0,00 \%$ \\
\hline 24 & 10176 & 11 & $0,00 \%$ & $100,0 \%$ & $9,09 \%$ & $0,00 \%$ \\
\hline 25 & 15711 & 14 & $64,29 \%$ & $100,0 \%$ & $42,86 \%$ & $7,14 \%$ \\
\hline 26 & 7598 & 60 & $45,00 \%$ & $98,3 \%$ & $50,00 \%$ & $1,67 \%$ \\
\hline 27 & 2192 & 10 & $40,00 \%$ & $80,0 \%$ & $20,00 \%$ & $0,00 \%$ \\
\hline Total & 229906 & 1739 & $34,50 \%$ & $67,40 \%$ & $38,53 \%$ & $2,13 \%$ \\
\hline
\end{tabular}


El tema más frecuentemente comentado en los tuits publicados durante el mes de mayo se refiere a las elecciones europeas, constituyendo el $22,6 \%$ de todos los mensajes. A continuación le siguen, por este orden: mensajes sobre cultura $(17,2 \%)$, de carácter social $(15,1 \%)$, deportes $(10,8 \%)$ y local $(7,6 \%)$. Tecnología y medioambiente constituyen temáticas que apenas son abordadas en estos mensajes.

Tabla 2. Distribución de los tuits y retuits de los alcaldes, mensajes retuiteados por otros usuarios y favoritos respecto al total de tuits por cada temática abordada. Totales y porcentajes. Fuente: elaboración propia.

\begin{tabular}{|l|c|c|c|c|c|c|c|c|}
\hline \multirow{2}{*}{ Temática } & \multicolumn{2}{|c|}{ Tuits } & \multicolumn{2}{c|}{$\begin{array}{c}\text { Retuits que } \\
\text { hacen los } \\
\text { alcaldes }\end{array}$} & $\begin{array}{c}\text { Retuiteados por } \\
\text { otros usuarios }\end{array}$ & \multicolumn{2}{c|}{ Favoritos $^{2}$} \\
\cline { 2 - 9 } & Totales & $\begin{array}{c}\text { \% } \\
\text { verticales }\end{array}$ & Totales & $\%$ & Totales & $\%$ & Totales & $\%$ \\
\hline Cultura & 299 & $17,19 \%$ & 78 & $26,09 \%$ & 222 & $74,25 \%$ & 199 & $66,56 \%$ \\
\hline Deportes & 187 & $10,75 \%$ & 72 & $38,50 \%$ & 128 & $68,45 \%$ & 128 & $68,45 \%$ \\
\hline Economía & 148 & $8,51 \%$ & 45 & $30,41 \%$ & 101 & $68,24 \%$ & 91 & $61,49 \%$ \\
\hline Educación & 25 & $1,44 \%$ & 2 & $8,00 \%$ & 15 & $60,00 \%$ & 14 & $56,00 \%$ \\
\hline $\begin{array}{l}\text { Elecciones } \\
\text { europeas }\end{array}$ & 393 & $22,60 \%$ & 195 & $49,62 \%$ & 231 & $58,78 \%$ & 195 & $49,62 \%$ \\
\hline Institucional & 113 & $6,50 \%$ & 29 & $25,66 \%$ & 63 & $55,75 \%$ & 52 & $46,02 \%$ \\
\hline Local & 133 & $7,65 \%$ & 51 & $38,35 \%$ & 106 & $79,70 \%$ & 83 & $62,41 \%$ \\
\hline Medioambiente & 19 & $1,09 \%$ & 3 & $15,79 \%$ & 15 & $78,95 \%$ & 16 & $84,21 \%$ \\
\hline Política & 64 & $3,68 \%$ & 19 & $29,69 \%$ & 41 & $64,06 \%$ & 40 & $62,50 \%$ \\
\hline Tecnología & 15 & $0,86 \%$ & 6 & $40,00 \%$ & 9 & $60,00 \%$ & 8 & $53,33 \%$ \\
\hline Social & 263 & $15,12 \%$ & 75 & $28,52 \%$ & 199 & $75,67 \%$ & 187 & $71,10 \%$ \\
\hline Otros & 80 & $4,60 \%$ & 26 & $32,50 \%$ & 42 & $52,50 \%$ & 37 & $46,25 \%$ \\
\hline
\end{tabular}

Prácticamente la mitad de los mensajes relativos a las elecciones europeas registrados en las cuentas analizadas son retuits de los alcaldes, situándose este porcentaje en torno al $40 \%$ para los mensajes de tecnología, deportes y locales. Por el contrario, la gran mayoría de los tuits sobre educación (92\%) y medioambiente (85\%) son mensajes creados por los propios representantes municipales; seguidos por los temas culturales $(74 \%)$ y políticos $(70 \%)$.

Los mensajes retuiteados en mayor proporción por los visitantes de las cuentas de los alcaldes, según la temática abordada, corresponden a medioambiente, cultura y temas sociales, por encima del $60 \%$; y los menos difundidos son los correspondientes a elecciones europeas $(42,2 \%)$.

Centrándonos en el número de veces que los mensajes son retuiteados, la media más elevada corresponde a deportes (14) y cultura (10). No obstante, para evitar la influencia de la dispersión de los datos y, más concretamente de casos extremos que desvían el valor de la media, desvirtuándola -como ocurre con las 768 cuentas que han retuitado

${ }^{1}$ Los datos corresponden a los mensajes que han sido retuiteados, independientemente del número de veces. Porcentajes horizontales.

${ }^{2}$ Los datos de favoritos corresponden a los mensajes que han sido marcados como favoritos, sin tener en cuenta el número de veces que hayan sido seleccionados. Porcentajes horizontales. 
un mensaje de carácter cultural-, utilizamos la mediana o el valor central del conjunto de datos de la variable. En la tabla 3 se puede observar que los temas típicos que son más difundidos por los usuarios de las cuentas corresponde a medioambiente, siendo otros, institucional, local, educación, economía y política los menos difundidos.

Tabla 3. Media, mediana, máximo y desviación típica (SD) del número de mensajes retuiteados, marcados como favoritos por otros usuarios y comentarios de la ciudadanía por temática. Fuente: elaboración propia

\begin{tabular}{|c|c|c|c|c|c|c|c|c|c|c|c|c|}
\hline \multirow[b]{2}{*}{ Temática } & \multicolumn{4}{|c|}{ Retuiteados } & \multicolumn{4}{|c|}{ Favoritos } & \multicolumn{4}{|c|}{ Comentarios de la ciudadanía ${ }^{3}$} \\
\hline & Media & $\begin{array}{c}\text { Media- } \\
\text { na }\end{array}$ & $\begin{array}{c}\text { Máxi- } \\
\text { mo }\end{array}$ & SD & Media & $\begin{array}{c}\text { Media- } \\
\text { na }\end{array}$ & Máximo & SD & Total & Media & $\begin{array}{c}\text { Máxi- } \\
\text { mo }\end{array}$ & SD \\
\hline Cultura & 23 & 2 & 3282 & 197 & 24 & 2 & 3851 & 244 & 375 & 1 & 19 & 3 \\
\hline Deportes & 17 & 4 & 698 & 68 & 13 & 4 & 262 & 34 & 294 & 2 & 25 & 4 \\
\hline Economía & 5 & 2 & 72 & 9 & 3 & 1 & 31 & 4 & 212 & 1 & 19 & 3 \\
\hline Educación & 2 & 1 & 8 & 3 & 1 & 1 & 4 & 1 & 38 & 2 & 9 & 3 \\
\hline $\begin{array}{l}\text { Elecciones } \\
\text { europeas }\end{array}$ & 6 & 2 & 275 & 19 & 4 & 1 & 102 & 9 & 338 & 1 & 19 & 3 \\
\hline Institucional & 2 & 1 & 13 & 2 & 1 & 1 & 7 & 1 & 118 & 1 & 14 & 3 \\
\hline Local & 3 & 2 & 28 & 4 & 2 & 1 & 16 & 3 & 123 & 1 & 16 & 2 \\
\hline Medioambiente & 3 & 3 & 8 & 2 & 2 & 2 & 5 & 1 & 26 & 1 & 6 & 2 \\
\hline Política & 9 & 4 & 117 & 21 & 4 & 2 & 51 & 9 & 159 & 2 & 44 & 7 \\
\hline Tecnología & 3 & 2 & 12 & 4 & 3 & 2 & 9 & 3 & 10 & 1 & 7 & 2 \\
\hline Social & 13 & 4 & 907 & 61 & 7 & 2 & 297 & 23 & 658 & 3 & 58 & 7 \\
\hline Otros & 4 & 1 & 166 & 19 & 3 & 1 & 91 & 12 & 99 & 1 & 22 & 3 \\
\hline
\end{tabular}

Desde la perspectiva de los usuarios que visitan las cuentas de estos representantes municipales (tabla 2), los temas favoritos con respecto al total de tuits sobre el tema corresponden a medioambiente y social. Sin embargo, si consideramos el número de veces que han sido marcados como favoritos, la media y mediana de mayor valor corresponde a deportes (tabla 3 ).

El grado de interactividad conseguida por los alcaldes mediante el uso de Twitter se ha obtenido utilizando como indicadores, por un lado, las respuestas de los seguidores a los comentarios o tuits remitidos por los usuarios principales $\mathrm{y}$, por otro, teniendo en cuenta las respuestas de los usuarios principales de las cuentas analizadas (máximos representantes municipales) a los comentarios de los seguidores, pudiendo hablar en este caso de un verdadero diálogo de los representantes municipales con la ciudadanía.

Del total de tuits publicados por los alcaldes, $557(32,02 \%)$ han generado 2.368 respuestas de la ciudadanía; de las cuales casi la mitad, el $48,7 \%$, corresponde a quejas de los ciudadanos, el 10,43\% incluyen preguntas, el 7,39\% contienen sugerencias, el $2,66 \%$ son quejas/sugerencias y el $2,58 \%$ refieren información; mientras que un $13,47 \%$ incluyen comentarios positivos y el $14,74 \%$ neutros.

Por temáticas, el mayor volumen de comentarios corresponde a temas sociales, culturales, elecciones europeas y deportes; los que menos interés generan son tecnología, medioambiente y educación. Si consideramos el conjunto de tuits publicados, la media

3 Los datos de la mediana no se incluyen ya que, dada la baja proporción de comentarios de los usuarios en relación con los tuits publicados, su valor es igual a 0. En su lugar, se incluyen los datos totales que no fueron incluidos en tablas anteriores. 
de respuestas por tuit más elevada corresponde a mensajes sobre temas sociales y seguido por política -también son los que experimentan una dispersión más elevada en el número de comentarios-; muy por detrás se sitúan deportes y economía. Con una media inferior a un comentario por tuit tendríamos tecnología y elecciones europeas.

Para finalizar, analizamos el número de respuestas de los alcaldes a los comentarios de los usuarios (quejas, sugerencias, informaciones, quejas/sugerencias, preguntas, comentarios neutros y positivos). En total se han contabilizado un total de 53 comentarios de los usuarios (de los 2.368 emitidos a 557 tuits) que han obtenido 73 respuestas de los alcaldes, cifra que nos indica el alto porcentaje de comentarios que quedan sin responder por parte de los alcaldes aun si consideráramos el carácter colectivo de muchas de estas contestaciones.

Los comentarios que incorporan quejas de los usuarios han generado la mayor cantidad de respuestas (49), seguidos por preguntas (35), comentarios positivos (34), sugerencias (33), comentario neutro (22), quejas/sugerencias (21) y, finalmente, información (9).

Por temáticas, deportes (22) es la que más respuestas genera por parte de los alcaldes. Muy por detrás se sitúan: social (12); local (8); economía (7); educación (6); elecciones europeas (5); institucional, política y cultura (3); medioambiente y otros (2) y tecnología no genera ninguno. En definitiva, un porcentaje muy elevado de preguntas y quejas formuladas por los ciudadanos quedan sin respuesta.

Tabla 4. Resumen comparativo de los temas más abordados por los alcaldes titulares de la cuenta (\% sobre total), temas más retuiteados, favoritos de los usuarios (mediana ordenada por media) y temas que generan más comentarios de los usuarios

(media). Fuente: Elaboración propia

\begin{tabular}{|l|l|l|l|}
\hline $\begin{array}{c}\text { Temas más } \\
\text { comentados por } \\
\text { alcaldes }\end{array}$ & $\begin{array}{c}\text { Temas más } \\
\text { retuiteados por los } \\
\text { ciudadanos } \\
\text { (mediana) }\end{array}$ & $\begin{array}{c}\text { Favoritos } \\
\text { marcados por los } \\
\text { ciudadanos } \\
\text { (mediana) }\end{array}$ & $\begin{array}{c}\text { Temas que generan } \\
\text { más comentarios } \\
\text { de los ciudadanos } \\
\text { (media) }\end{array}$ \\
\hline Europeas $(22,6 \%)$ & Deporte (4) & Deportes (4) & Deportes (2) \\
\hline Cultura $(17,2 \%)$ & Social (4) & Cultura (2) & Política (2) \\
\hline Social $(15,1 \%)$ & Política (4) & Política (2) & Cultura (2) \\
\hline Deportes $(10,8 \%)$ & Medioambiente (3) & Social (2) & Educación (2) \\
\hline Economía $(8,5 \%)$ & Cultura (2) & Tecnología (2) & Otros (1) \\
\hline Local $(7,7 \%)$ & Europeas (2) & Europeas (1) & Institucional (1) \\
\hline Institucional $(6,5 \%)$ & Economía (2) & Otros (1) & Local (1) \\
\hline Otros $(4,6 \%)$ & Local (2) & Economía (1) & Europeas (1) \\
\hline Política $(3,7 \%)$ & Tecnología (2) & Local (1) & Tecnología (1) \\
\hline Educación $(1,4 \%)$ & Otros (1) & Medioambiente (2) & Medioambiente (1) \\
\hline Medioambiente $(1,1 \%)$ & Educación (1) & Educación (1) & Social (1) \\
\hline Tecnología $(0,9 \%)$ & Institucional (1) & Institucional (1) & Economía (1) \\
\hline
\end{tabular}

En la tabla 4 podemos observar la divergencia entre los temas más comentados por los alcaldes y los más retuiteados, favoritos y comentados por los seguidores de los anteriores. Cabe resaltar que el alto interés que las elecciones al Parlamento Europeo generan entre los alcaldes no es compartido en la misma medida por los usuarios, así si este tema genera la mayor cantidad de tuits de los alcaldes, su posición en referencia al resto de temáticas entre los mensajes difundidos, marcados como favoritos o comentados por los usuarios queda muy postergado. No cabe duda, los depor- 
tes son los temas que más interés suscitan entre los usuarios de las cuentas analizadas; seguidos por cultura, política y social. Cabe resaltar que los mensajes sobre medioambiente remitidos por los alcaldes son los que, en términos generales, resultan más difundidos por los ciudadanos que siguen las cuentas de los alcaldes.

\section{Conclusiones y discusión}

Partiendo de la premisa de que las capitales de provincias y ciudades autonómicas actúan como referentes en cada una de sus respectivas circunscripciones provinciales, en este trabajo se analizan las prácticas de sus representantes municipales a través de Twitter. En particular, se buscaba comprobar si, mediante el uso de esta herramienta de microblogging, los alcaldes incentivan la participación y la comunicación con sus ciudadanos, contribuyendo así a mejorar la salud del sistema democrático.

Los primeros resultados de la revisión de los perfiles abiertos en Twitter nos permiten confirmar que los representantes municipales de las capitales de provincia y ciudades autonómicas no están aprovechando la disponibilidad de este canal de participación ciudadana. Únicamente dos terceras partes de la muestra disponen de un perfil en Twitter y apenas la mitad mantienen también las cuentas activas durante el periodo investigado.

La apertura de una cuenta en Twitter, sin embargo, no presupone una intencionalidad de fomentar la interactividad. A excepción de determinados casos aislados, la media de mensajes publicados resulta muy baja y ello a pesar de la coincidencia en el mes investigado con las elecciones europeas que, como se ha comprobado, han generado un importante porcentaje de la actividad ejercida por los alcaldes. Este limitado uso que las máximas autoridades municipales están haciendo de esta red social demuestra el exiguo interés por mantener una comunicación activa con sus ciudadanos a través de esta vía.

Ahondando en este punto, algo más de un tercio de los mensajes no son sino retuits que los alcaldes hacen de los tuits de otras cuentas. Uno de los alcaldes, incluso, no ha generado ningún mensaje propio. Por el contrario, el 50\% de los mensajes emitidos por los alcaldes son difundidos por los usuarios en otras cuentas.

Aunque no se ha analizado explícitamente, los datos alcanzados nos permiten intuir una proporcionalidad entre la voluntad de los alcaldes por interactuar con los vecinos y la mayor participación de éstos, haciéndose patente el carácter dinamizador de Twitter al favorecer el diálogo entre representantes y representados.

Uno de los motivos que puede alentar a los representantes locales para fomentar la comunicación con la ciudadanía es el predominio de quejas que se plantean como respuesta a los tuits emitidos y que, unidas a las sugerencias, engloban más de la mitad de sus comentarios. Estas contribuciones de los ciudadanos, cuando se aportan como críticas constructivas, pueden servir de incentivo para la actualización de la agenda política, de los proyectos e iniciativas que los alcaldes y sus equipos de gobierno tengan previstos y desarrollen en sus respectivos municipios. Si bien los comentarios positivos también pueden suponer un acicate, no resultan tan productivos para la mejora de la gestión diaria de un ayuntamiento como el enriquecimiento que proporciona otros puntos de vista distintos ofrecidos por los ciudadanos, preocupados igualmente por el futuro y avance de su entorno. 
El uso que realizan los alcaldes de Twitter dista de la potencialidad de esta red para generar un verdadero y efectivo marco de interactividad con sus seguidores. La importante cantidad de comentarios publicados por estos últimos contrasta notablemente con las escasas contestaciones que reciben de los representantes municipales, quienes utilizan el microblogging de forma similar a un escaparate público, como un bando o un periódico oficial en el que exponen sus iniciativas, logros, experiencias en la gestión o acciones en el municipio, sin aportar una respuesta a las inquietudes expresadas por los usuarios; en otras palabras: sin entablar un diálogo que incremente en el ciudadano un sentimiento de que está siendo escuchado por sus representantes.

Este extremo no solo queda patente en la cantidad de respuestas; la temática preferida por los usuarios para publicar sus comentarios no coincide con los contenidos que proponen los representantes locales en sus tuits. La marcada difusión de tuits referidos a las elecciones europeas ofrecen un nítido ejemplo: no tienen un hondo calado entre los ciudadanos, los cuales se decantan por informaciones de otros ámbitos, principalmente y por este orden, las relacionadas con deportes, política, cultura y educación.

Los resultados hasta aquí obtenidos invitan a crear un marco de reflexión sobre la potencialidad de Internet y, en particular de Twitter, para generar entre ciudadanos y representantes municipales una interactividad de igual a igual que incentive la participación de los primeros en el desarrollo y avance del municipio, a la vez que los segundos elaboren agendas políticas que coincidan en mayor grado con las inquietudes, demandas y necesidades de sus representados, actualizadas en periodos de tiempo más frecuentes que los cuatrienios de cada legislatura.

Es pertinente también profundizar en las causas que provocan la práctica inexistencia de una constante y deseable red de comunicación entre políticos y ciudadanos que, a pesar de tener los medios eficaces que aportan las nuevas tecnologías, no son utilizados de forma provechosa por ninguna de las dos partes. La escasa implicación en la política que se muestra en la mayor parte de la ciudadanía puede ser un factor influyente, aunque es seguro que no es el único si se parte de la base de que las administraciones públicas están obligadas a incentivar y potenciar la participación.

\section{Referencias bibliográficas}

AMMANN, Sky L. (2010): "Why Do They Tweet? The Use of Twitter by U.S. Senate Candidates in 2010". Social Science Research Network: http://ssrn.com/abstract=1725477 o http://dx.doi.org/10.2139/ssrn.1725477 [Consulta: 29 de mayo de 2014].

ARROYO, Luis (2012): "10 razones por las que Twitter no sirve para (casi) nada en política", en ARROYO's blog: http://www.luisarroyo.com/2012/05/06/10-razonespor-las-que-twitter-no-sirve-para-casi-nada-en-politica/ [Consulta: 20 de julio de 2014].

ASOCIACIÓN PARA LA INVESTIGACIÓN DE MEDIOS DE COMUNICACIÓN (AIMC) (2014): “Navegantes en la Red": http://download.aimc.es/aimc/J5d8yq /macro2013.pdf [Consulta: 29 de mayo de 2014]. 
BARBERÁ, Pablo y RIVERO, Gonzalo (2012): “¿Un tweet, un voto? Desigualdad en la discusión política en Twitter", en I Congreso Internacional en Comunicación Política y Estrategias de Campaña. Madrid, 6-7 de julio. http://www.alice-comunicacionpolitica.com/files/ponencias/58-4ffff91581342177169-ponencia-1.pdf [Consulta: 20 de mayo de 2014].

BOLLEN, Johan et al. (2011): "Twitter mood predicts the stock market", en Journal of Computational Science. Journal of Computational Science, 2 (1), pp. 1-8.

BRUGUÉ, Quim et al. (2005): "Participación y democracia: asociaciones y poder local", en Jornadas Cómo construir una ciudad participativa. Málaga, 5-7 de octubre. http://www.uimunicipalistas.org/xcongreso/documentacion/documentos /V/8.pdf [Consulta: 20 de mayo de 2014].

CALDEVILLA, David (2009): "Democracia 2.0: La política se introduce en las redes sociales", en Pensar la publicidad: revista internacional de investigaciones publicitarias. 3(2), pp. 31-48.

CASTELLÓ, Araceli et al. (2014): "Twitter como canal de comunicación corporativa y publicitaria", en Communication \& Society /Comunicación y Sociedad, 27(2), pp. 21-54.

CONGOSTO, Ma Luz et al. (2011): “Twitter y política: Información, opinión y ¿Predicción?", en Cuadernos de Comunicación Evoca, 4, pp. 11-15.

CONSEJO DE EUROPA (2001): La participación de los ciudadanos en la vida pública local. Recomendación e informe explicativo del Comité de Ministros. http://www.seap.minhap.gob.es/dms/es/areas/politica_local/participacion-eell-aeuropeos/recomendacion.pdf [Consulta: 17 de mayo de 2014].

CONSTITUCIÓN ESPAÑOLA (1978).

CRAWFORD, Ewan (2009): "A new sort of democracy?: The opinion pages in the Scottish daily quality press". Journalism 10, no 4, p. 454.

CULVER, Sherri \& JACOBSON, Thomas (2012): "Media Literacy and Its Use as a Method to Encourage Civic Engagement", en Comunicar, vol. 20, no 39, p. 73-80. http://eprints.rclis.org/17753/1/en73-80.pdf [Consulta: 20 de mayo de 2014].

DAHLGREN, Peter (2005): "The Internet, public spheres, and political communication: Dispersion and deliberation", en Political communication, vol. 22, no 2, pp. 147-162.

DELTELL, Luis et al. (2013): "Predicción de tendencia política por Twitter: Elecciones andaluzas 2012", en Ámbitos, Revista Internacional de Comunicación, 22. $\mathrm{http}: / /$ ambitoscomunicacion.com/2013/prediccion-de-tendencia-politica-por-twitter-elecciones-andaluzas-2012/ [Consulta: 23 de mayo de 2014].

FERNÁNDEZ, Carmen Beatriz (2012): "Twitter y la ciberpolítica", en Anuario electrónico de estudios en Comunicación Social " Disertaciones", 5 (1). http://erevistas.saber.ula.ve/index.php/Disertaciones/article/viewFile/3490/3761 [Consulta: 24 de mayo de 2014]. 
FONT, Javier y PARRADO, Salvador. (2000): "Eligiendo socios en la administración municipal española: Los consorcios y las mancomunidades". Cuadernos de Gobierno y de Administración, 3, 15-30.

GONZÁLVEZ PÉREZ, Vicent y CONTRERAS PULIDO, Paloma (2014): “Empoderar a la ciudadanía mediática desde la educomunicación”, en Comunicar, vol. 21, no 42, pp. 129-136. http://rabida.uhu.es/dspace/bitstream/handle/10272/7759/empoderar_a_la_ciudadan\%C3\%ADa.pdf?sequence=2 [Consulta: 24 de mayo de 2014].

HAVICK, John (2000): "The impact of the Internet on a television-based society". Technology in Society, vol. 22, no 2, p. 273-287.

INTERACTIVE ADVERTISING BUREAU SPAIN (2014): "V Estudio Anual Redes Sociales". http://www.iabspain.net/wp-content/uploads/downloads/2014/04/V-Estudio-Anual-de-Redes-Sociales-versi\%C3\%B3n-reducida.pdf [Consulta: 21 de mayo de 2014].

JANOSCHKA, Michael (2011): "Geografías urbanas en la era del neoliberalismo. Una conceptualización de la resistencia local a través de la participación y la ciudadanía urbana". Investigaciones geográficas, vol. 76, pp. 118-132. http://www.scielo.org.mx/scielo.php?script=sci_arttext\&pid=S018846112011000300009 [Consulta: 21 de mayo de 2014].

JAVA, Akshay et al. (2007): "Why we twitter: understanding microblogging usage and communities", en Proceedings of the 9th WebKDD and 1st SNA-KDD 2007 workshop on Web mining and social network analysis, pp. 56-65. http://aisl.umbc.edu/resources/369.pdf [Consulta: 22 de mayo de 2014].

JEFATURA DEL ESTADO. Ley 7/1985, de 2 de abril, Reguladora de las Bases del Régimen Local. Boletín Oficial del Estado 80, 3 de abril de 1985, nº 80, p. 89458964.

KRUIKEMEIER, Sanne (2014): "How political candidates use Twitter and the impact on votes" en Computers in Human Behavior, n ${ }^{\circ}$ 34, pp. 131-139.

MANCERA, Ana y PANO, Ana (2013): El discurso político en Twitter. Barcelona, Anthropos Divulga.

MARGOLIS, Michael \& RESNICK, David (2000): Politics as usual. USA, Sage Publications.

METAXAS, Panagiotis Takis et al. (2011): "How (not) to predict elections". Privacy, security, risk and trust (PASSAT) IEEE third international conference on social computing (SocialCom), pp. 165-171.

PAK, Alexander \& PAROUBEK, Patrick (2010): “Twitter as a Corpus for Sentiment Analysis and Opinion Mining". LREC: http://incctps.googlecode.com/svn/trunk /TPFinal/bibliografia/Pak\%20and\%20Paroubek\%20(2010).\%20Twitter\%20as\%20 a $\% 20$ Corpus $\% 20$ for $\% 20$ Sentiment $\% 20$ Analysis $\% 20$ and $\% 20$ Opinion $\% 20$ Mining.pdf [Consulta: 22 de mayo de 2014]. 
PASTOR, Enrique (2009): Participación ciudadana y gestión de las políticas sociales municipales. Murcia, Universidad de Murcia, Editum, Vol. 10.

PINDADO, Fernando (2004): "La participación ciudadana en el ámbito local", en FEMP. Madrid, 2008. http://www.irun.org/down/ponencias_presupuestos/Ponencia-pintado.pdf [Consulta: 24 de mayo de 2014].

RODRÍGUEZ, Roberto; UREÑA, Daniel (2011): "Diez razones para el uso de Twitter como herramienta en la comunicación política y electoral". Comunicación y pluralismo, $\mathrm{n}^{\circ}$ 10, pp. 89-116: http://www.masconsulting.es/wpcontent/uploads/2012/05/Art_ComunicacionPluralismo.pdf [Consulta: 23 de mayo de 2014].

ROMERO, Daniel M. et al. (2011): "Differences in the mechanics of information diffusion across topics: idioms, political hashtags, and complex contagion on Twitter". Proceedings of the 20th international conference on World wide web,. ACM, pp. 695-704. http://leonidzhukov.net/hse/2011/seminar/papers/www11-hashtags.pdf [Consulta: 22 de mayo de 2014].

SECRETARÍA DE ESTADO DE COOPERACIÓN TERRITORIAL (2005): Libro Blanco para la Reforma del Gobierno Local. Madrid, Ministerio de Administraciones Públicas.

TORRES DA SILVA, Marisa (2013): "Online forums, audience participation and modes of political discussion: readers' comments on the Brazilian presidential election as a case study". Comunicación y Sociedad, vol. 26, nº. 4, pp. 175-193. http://dspace.unav.es/dspace/bitstream/10171/35567/1/20131028164821.pdf [Consulta: 20 de mayo de 2014].

TUMASJAN, Andranik et al. (2010): "Predicting Elections with Twitter: What 140 Characters Reveal about Political Sentiment". ICWSM, vol. 10, pp. 178-185. http://www.aaai.org/ocs/index.php/ICWSM/ICWSM10/paper/viewFile/1441/1852 [Consulta: 23 de mayo de 2014].

VARONA, David (2014): La influencia de las redes de Internet en las prácticas, hábitos y técnicas de los periodistas españoles. Madrid, Universidad Complutense. http://eprints.ucm.es/25351/1/T35344.pdf [Consulta: 29 de mayo de 2014]. 